\title{
ON THE CLASSIFICATION OF SOLUTIONS TO THE ZERO-SURFACE-TENSION MODEL FOR HELE-SHAW FREE BOUNDARY FLOWS
}

\author{
BY \\ S. RICHARDSON \\ Department of Mathematics and Statistics, University of Edinburgh, Scotland
}

\begin{abstract}
We add a possibility to the catalogue of scenarios given by Hohlov and Howison for the behavior of Hele-Shaw free boundary flows when surface tension is assumed to be absent: suction from an initially connected blob of fluid can result in regions of fluid being left marooned some distance from the suction point.
\end{abstract}

1. Introduction. In a recent paper, Hohlov and Howison [1] proposed a classification for solutions describing the evolution of the free boundary in a Hele-Shaw flow when the zero-surface-tension boundary condition is employed. We contemplate a fluid region that is initially bounded, connected, and simply-connected, and suppose the motion to be driven by either injection or suction at just one point within this region. We add a further possibility to the classification: suction may result in the connectivity of the region occupied by the fluid being destroyed, leaving islands of fluid, possibly infinite in number, isolated from the suction point.

The existence of such solutions follows simply from the time-reversible nature of the flows. Suppose we begin with a number of disjoint blobs of fluid and then inject at a point anywhere in the plane; it may be within a pre-existing blob, on the boundary of such a blob, or in a region initially devoid of fluid. It is easy to envisage such a situation where the consequent expansion will ultimately reach and absorb the outlying fluid regions. A reversal of this flow, substituting suction for injection, must then result in the restoration of the original distribution of fluid.

To follow this evolution analytically, and display explicit solutions showing this behaviour, it is convenient to exploit the Cauchy transform; see [3] for more details.

2. The Cauchy transform. Suppose the fluid at a given time to occupy a subset $D$ of the usual complex $z$-plane. To avoid complications that are not germane to the present discussion, we shall suppose $D$ to be bounded, but do not insist that it be connected so that it is not a domain in the usual technical sense. We may suppose it to be the

Received August 17, 1994.

1991 Mathematics Subject Classification. Primary 76S05, 76D99. 
union of disjoint domains and, again merely to avoid unnecessary complications here, we assume each disjoint component of $D$ to be simply-connected, and to be the interior of its closure. We also suppose the interior of the complement of $D$ to be connected, but not necessarily simply-connected; it contains a neighbourhood of infinity.

The Cauchy transform of $D$ is a function, dependent on $D$, that is defined and continuous in the entire complex $z$-plane. On the interior of the complement of $D$ it is an analytic function $h_{e}(z)$, and it is this portion of the Cauchy transform that is important; we may contemplate the analytic continuation of $h_{e}(z)$ into $D$ where it will possess singularities, but it is not there equal to the Cauchy transform of $D$.

If we begin with an empty Hele-Shaw cell and inject an area $\pi r^{2}$ at the point $z=a$, the resultant circular disc has an associated function

$$
h_{e}(z)=\frac{r^{2}}{z-a} .
$$

Now a crucial property of $h_{e}(z)$ is that it is additive for disjoint subsets of the plane. If we begin with an empty Hele-Shaw cell and inject at several points, the relevant $h_{e}(z)$ is just a sum of terms of the form (2.1); with only small areas injected, this corresponds to a disjoint union of circular discs, but the same sum continues to describe any situation reached as the discs grow and coalesce; we suppose the evolution does not result in any region occupied by fluid becoming multiply-connected, a technical difficulty that will be discussed in Sec. 5.

3. Examples. We can now give a simple, explicit example of the behaviour we seek to demonstrate. Consider

$$
h_{e}(z)=\frac{R^{2}}{z}+\frac{r^{2}}{z-a},
$$

where we may suppose $a$ to be real and positive. With $R=0$, this represents a circular disc of radius $r$ and centre $z=a$ produced, perhaps, by injection at $z=a$ as was (2.1). Suppose here that $r<a$, so that this disc does not cover the origin. Then injection of an area $\pi R^{2}$ at $z=0$ produces (3.1). As $R$ increases from 0 to $a-r$ it represents two disjoint circular discs; these coalesce when $R=a-r$, and further injection increasing $R$ involves a single, simply-connected blob. Reversal of this flow produces a suction problem with all the fluid eventually removed from around the suction point, but with an isolated circular disc of fluid left behind. A particular example is sketched in Fig. 1.

In one sense, the evolution under suction shown in Fig. 1 may be regarded as another manifestation of cusp formation; we have carefully arranged that two cusps should form at the same instant, coalescing as they do so and pinching off a region of fluid. However, as with one of the examples discussed by Hohlov and Howison [1], the development of the cusps does not here lead to blow-up in the usual sense; a perfectly acceptable solution persists beyond this time. Moreover, the appearance of cusps here is an artefact of our desire to produce an explicit analytic solution. We can easily envisage the injection problem corresponding to Fig. 1 with the circular disc on the right replaced, for example, by a body with a corner pointing towards the injection point. Reversal of this will produce a pinching-off of a different character. 


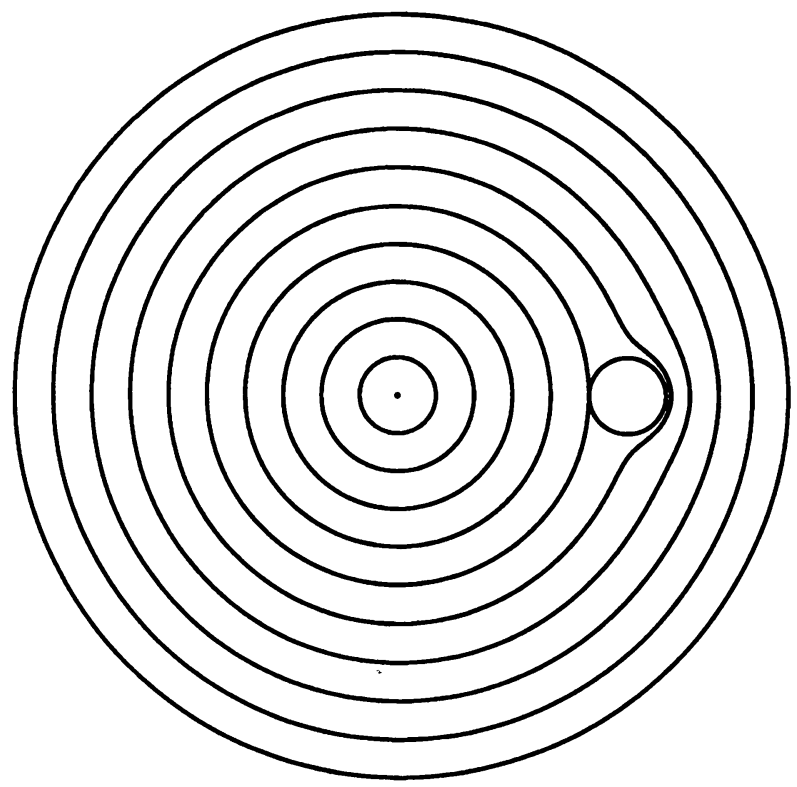

FIG. 1. The solution given by (3.1) with $a=1.2$ and $r=0.2$. The moving boundary is drawn for $R=0$ to $R=2$ at increments of 0.2 . Suction leaves behind a circular disc of fluid, but otherwise empties the cell.

It is clear that we could elaborate on the solution of Eq. (3.1) and Fig. 1 in a number of ways. For example, adding further simple poles to (3.1) will produce solutions with any number, finite or infinite, of circular discs left behind following suction. However, (3.1) itself may also be exploited in a different direction.

Consider (3.1) with $R=0$, and the second term produced by injecting an area $\pi r^{2}$ at $z=a$ but now with $r>a$, so that this initial circular disc covers the origin. We may now consider suction at the origin; with $\pi R^{2}$ the area injected at $z=0$, this simply requires us to allow $R^{2}$ to be negative in (3.1). This problem of injection or suction in an initial circular disc was presented as an example in [2]. With suction (and $a \neq 0$ ), a cusp develops in the moving boundary and the solution blows up when $R^{2}=R_{0}^{2}$, where $R_{0}^{2}$ is the unique negative root of a cubic equation in $R^{2}$, specifically

$$
\left(2 R^{2}+2 r^{2}+a^{2}\right)^{3}=27 a^{2}\left(r^{2}-R^{2}\right)^{2} .
$$

This equation does not appear in [2], but is easily deduced from the formulae given therein.

Incorporating this solution into our scheme, we can obtain further explicit solutions showing other features. Consider, for example, the function

$$
h_{e}(z)=\frac{R^{2}-R_{0}^{2}}{z}+\sum_{k=1}^{n} \frac{r_{k}^{2}}{z-a_{k}},
$$




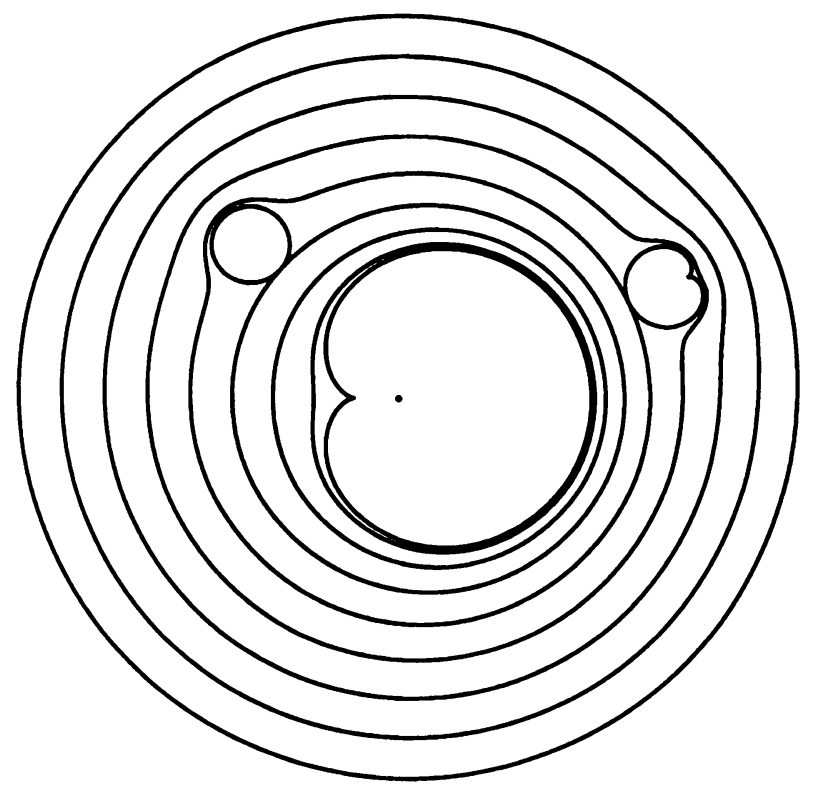

FIG. 2. The solution given by (3.3) with the values appearing in (3.4). The moving boundary is drawn for $R=0$ to $R=24$ in increments of 3. Suction first leaves behind a blob with a cusp and then a circular disc of fluid. The solution then blows up as a cusped body forms about the suction point at the origin, marked with a dot.

where

$$
\left.\begin{array}{c}
n=4, \quad R_{0}=9, \\
a_{1}=2, \quad a_{2}=-10+6 \sqrt{3} i, \quad a_{3}=18.7+8 i, \quad a_{4}=18.3+7.82 i, \\
r_{1}^{2}=175, \quad r_{2}^{2}=7, \quad r_{3}^{2}=-8.4656, \quad r_{4}^{2}=15.5844 .
\end{array}\right\}
$$

The motion produced by this for $R$ between 0 and 24 is as in Fig. 2 .

For $R=0$ and the data in (3.4), the first term in (3.3) and the term with $k=1$ in the sum correspond to the larger cusped body about the suction/injection point at $z=0$; it is based on the solution $(r / a)^{2}=175 / 4$ and $(R / a)^{2}=-81 / 4$ of (3.2). The term with $k=2$ in the sum corresponds to the circular disc. The terms with $k=3$ and $k=4$ in the sum correspond to the smaller cusped body; it is based on the solution $(r / a)^{2}=81$ and $(R / a)^{2}=-44$ of $(3.2)$.

Injection at the origin of an area $\pi R^{2}$ first affects only the cusped body about the origin as $R$ increases. At $R=R_{0}=9$, this body becomes circular and simultaneously hits and absorbs the circular blob, but the smaller cusped body remains unaffected. This is hit and absorbed in turn at $R=12$, and for $R>12$ the fluid occupies a single, simplyconnected region. A reversal of this flow by suction then results in a fragmentation of this region, followed by a blow-up. Note that here three cusps form at the instant the cusped body is left behind, but only two take part in the pinching-off process.

The figures in (3.4) that produce the above effects, with all the interesting behaviour occurring at values of $R$ that are integer multiples of 3 and the two bodies precisely 
cusped, are exact except for the number 18.7 in $a_{3}$-and the related number 18.3 in $a_{4}$, though the difference $a_{3}-a_{4}=0.4+0.18 i$ is exact. Changing this 18.7 moves the smaller cusped body, and it was determined visually so that the free boundary just touches this body when $R=12$.

It is evident that somewhat more complicated solutions could be constructed. Indeed, since any simply-connected body can be approximated to arbitrary accuracy by a body whose $h_{e}(z)$ consists only of simple poles, the present methods have wide applicability. Moreover, one may deal exactly with more general bodies by exploiting other forms of $h_{e}(z)$ for which we can obtain solutions. We note that the shapes obtained using quadratic and cubic maps in [1] correspond to $h_{e}(z)$ involving double and triple poles, respectively; the cardioid appearing in [1] is a degenerate form of the cusped curves appearing here, with the two simple poles in (3.1) allowed to coalesce into a double pole in a manner familiar from classical inviscid hydrodynamics. Other cases given in [1] are special instances of those considered here, and the present approach makes the algebra somewhat more transparent and motivates the form these solutions take. For example, Fig. 4 in [1] is the special case $r=a$ of (3.1), while Fig. 6 results if we take $n=2$, $R_{0}=0$, and $r_{1}=r_{2}=a_{1}=-a_{2}$ (real and positive) in (3.3). One may also deal with $h_{e}(z)$ having logarithmic branch cuts (which may be produced by injecting along slits) as shown in [3].

4. The conformal maps. The previous section concentrated solely on the function $h_{e}(z)$ because of the crucial role it plays, but we must still show how the geometric configurations, as displayed in Figs. 1 and 2, for example, can be determined from $h_{e}(z)$. However, since an effective routine for doing this was given in [2], we here merely recall the essentials as they pertain to the present situation.

Suppose we have an $h_{e}(z)$ of the form (3.3) that we know corresponds to a connected, simply-connected blob. For example, in Fig. 2 we have $n=1$ for $0<R<9, n=2$ for $9<R<12$, and $n=4$ for $R>12$ if we are simply following the behaviour of that connected region occupied by fluid that contains the injection/suction point at the origin.

Let $z=f(\zeta)$ map the unit disc $|\zeta|<1$ conformally onto this region; it is convenient here to require that $f(0)=0$, as we may. Then the properties of the Cauchy transform imply that

$$
h_{e}(f(\zeta))-\bar{f}(1 / \zeta) \text { is analytic for }|\zeta|<1,
$$

where $\bar{f}(\zeta)$ denotes the analytic function conjugate to $f(\zeta)$; that is, $\bar{f}(\bar{\zeta})=\overline{f(\zeta)}$. Equation (3.3) then implies that $\bar{f}(1 / \zeta)$ must have the form

$$
\bar{f}(1 / \zeta)=\frac{\alpha}{\zeta}+\sum_{k=1}^{n} \frac{\beta_{k}}{\zeta-\gamma_{k}}
$$

for some constants $\alpha, \beta_{k}$, and $\gamma_{k}$ with $\left|\gamma_{k}\right|<1$, whence

$$
f(\zeta)=\bar{\alpha} \zeta+\sum_{k=1}^{n} \frac{\bar{\beta}_{k} \zeta}{1-\bar{\gamma}_{k} \zeta}
$$


Ensuring the cancellation of the apparent simple poles in $|\zeta|<1$ of the two terms in (4.1), we obtain the equations

$$
\alpha f^{\prime}(0)=R^{2}-R_{0}^{2}, \quad f\left(\gamma_{k}\right)=a_{k}, \quad \beta_{k} f^{\prime}\left(\gamma_{k}\right)=r_{k}^{2} \quad \text { for } k=1,2, \ldots, n .
$$

These are $2 n+1$ complex equations to determine the $2 n+1$ complex parameters in (4.2), and the problem has been reduced to the solution of a system of algebraic equations.

Some thought implies that everything cannot be quite as straightforward as its seems, for we know that we should also be able to specify $\arg \left\{f^{\prime}(0)\right\}$. In fact, the equations in (4.3) are not independent; if we have any solution for the $2 n+1$ parameters $\alpha, \beta_{k}$, and $\gamma_{k}$, then multiplying them all by the same constant of unit modulus produces another. The imaginary part of the first equation is a consequence of the others and can be discarded. The standard normalization with $f^{\prime}(0)>0$ can then be imposed, and is equivalent to taking $\alpha$ to be real and of the same sign as $R^{2}-R_{0}^{2}$. (If $R^{2}-R_{0}^{2}=0$ then $\alpha=0$, but this special case is much simpler with an exact solution in the context of Fig. 2.)

At first sight, the change in the form of the mapping in Fig. 2 as $R$ passes through the values $R=9$ and $R=12$ may seem curious, for the Carathéodory kernel theorem implies that the mapping function $f(\zeta)$ must be a continuous function of $R$ for $\zeta$ confined to a compact subset of $|\zeta|<1$ if we maintain $f^{\prime}(0)>0$ throughout. In fact, considering the injection problem, as $R$ increases through a value where $n$ in (4.2) increases, the new poles are born on the unit circle $|\zeta|=1$ with zero residue, while the coefficients involved in the already-existing poles vary in a continuous manner; so the conclusions of this theorem are respected.

If $h_{\epsilon}(z)$ should have poles of order greater than one, or possess logarithmic branch points as in [3], a similar solution procedure to the above is available. The form of the mapping can be written with unknown coefficients, and these coefficients are then determined by solving a system of equations similar to (4.3).

5. Concluding remarks. At the end of Sec. 3 we indicated how the present approach can be exploited to furnish more general solutions. However, it should be pointed out that the shapes of the blobs left behind as the main blob retreats under suction cannot be completely arbitrary, there being restrictions imposed by the curious role played by multiple-connectivity in such problems. Suppose, for example, that we contemplate injection as envisaged in Fig. 2, but turn the small cusped body through $180^{\circ}$. (This may be effected by interchanging the values for $r_{33}^{2}$ and $r_{4}^{2}$ in (3.4).) The advancing boundary will then trap air in a region near the cusp. There is just one orientation of this body for which the moving boundary hits it simultaneously at two points, but for a range of orientations either side of this, the evolution will also lead to the formation of an air pocket whose properties must then be considered. If we make the assumption that this air is incompressible (so that the air hole must stay a constant area) there appears to be no solution consistent with our model beyond the instant when the hole is formed. On the other hand, if we suppose the air within the hole to be maintained at the same pressure as that at other points devoid of fluid, then a solution does exist with the air hole decreasing in area and eventually vanishing. But now we run into difficulties when we try to reverse this process in an attempt to obtain a suction problem that leaves 
the cusped body with this orientation behind. As the suction proceeds through the configuration where the hole disappeared during injection, the blob must be provoked to instigate the growth of the hole at the correct place. Even if this can be arranged, we are then faced with a greater difficulty, for such problems involving the growth of a hole from a point appear to be ill-posed: uniqueness fails. A more detailed discussion of such subtleties, arising in a particular situation involving a doubly-connected region of fluid, can be found in [4].

Acknowledgment. The numerical computations required for this paper were performed using Mathematica (Version 2), and its graphics facilities were also employed for producing the figures.

\section{REFERENCES}

[1] Y. E. Hohlov and S. D. Howison, On the classification of solutions to the zero-surface-tension model for Hele-Shaw free boundary flows, Quart. Appl. Math. 51, 777-789 (1993)

[2] S. Richardson, Hele Shaw flows with a free boundary produced by the injection of fluid into a narrow channel, J. Fluid Mech. 56, 609-618 (1972)

[3] S. Richardson, Hele Shaw flows with time-dependent free boundaries involving injection through slits, Stud. Appl. Math. 87, 175-194 (1992)

[4] S. Richardson, Hele-Shaw flows with time-dependent free boundaries in which the fluid occupies a multiply-connected region, European J. Appl. Math. 5, 97-122 (1994) 